\title{
Clinical Profile of Children with Acute Febrile Encephalopathy in a Tertiary Health Care Center of Nepal
}

\author{
Sharma $\mathrm{P}^{1}$, Sarmah $B K^{2}$, Kayastha $\mathrm{P}^{3}$, Shrestha $\mathrm{A}^{4}$, Tiwari $\mathrm{D}^{5}$
}

\begin{abstract}
Introduction: Acute febrile encephalopathy is a common and severe neurological syndrome that is associated with significant morbidity and mortality in children. The etiologies differ according to geographical regions and appropriate and efficient protocols for investigations and management requires proper understanding of various potential etiologies. Material and Methods: In this prospective observational study, 54 children of one month to 14 years presenting to the College of Medical Sciences, Bharatpur, Nepal with acute onset of fever with altered sensorium were clinically evaluated and investigated. Clinical examinations included temperature, pulse rate, heart rate, blood pressure, coma severity by GCS, respiratory pattern and detail systemic and CNS examination. Etiology of acute febrile encephalopathy was based on clinical history, examination and relevant laboratory investigations. Results: The incidence of acute febrile encephalopathy was $5.5 \%$ of the total hospital admissions. There were $35(64.8 \%)$ boys and $19(35.2 \%)$ girls. The maximum number of children $24(44.4 \%)$ were within the age group of one to five years of age. The most common presenting complains besides fever and altered sensorium were seizures and vomiting which was present in $59.3 \%$ and $46.3 \%$ of children respectively. Signs of meningeal irritation, hypertonia, brisk deep tendon reflexes and extensor plantar response was present in $50 \%, 24 \%, 29.6 \%$, and $44.4 \%$ respectively. The diagnosis based on clinical findings and laboratory investigations were viral encephalitis 34(63\%), bacterial meningitis $12(22.2 \%)$, cerebral malaria $4(7.4)$, enteric encephalopathy $3(5.6 \%)$ and hepatic encephalopathy $1(1.9 \%)$. Conclusion: Viral encephalitis and bacterial meningitis are the most common cause of acute febrile encephalopathy. Preventive strategies can be undertaken to decrease the incidence.
\end{abstract}

Key words: Bacterial meningitis, encephalopathy, incidence, viral encephalitis

\section{Introduction}

Encephalopathy is a diffuse disease of brain that alters its - structure and function ${ }^{1}$. Acute febrile encephalopathy is defined as short duration fever with altered consciousness which is a common problem in pediatric patients leading to hospitalization ${ }^{2}$.
'Dr. Poonam Sharma, MBBS, MD, Department of Paediatrics, Shahid Gangalal National Heart Center, Kathmandu, Nepal, ${ }^{2}$ Dr. BK Sarmah, MBBS. MD, Professor and HoD, Department of Paediatrics, College of Medical Sciences, Bharatpur, Chitwan, Nepal, 'Dr. PawanaKayastha, MBBS. MD, Department of Paediatrics, Kathmandu Medical College, Kathmandu, Nepal, ${ }^{4}$ Dr. Asim Shrestha, MBBS. MD. Department of Paediatrics, Kathmandu University School of Medical Sciences, Dhulikhel, Nepal, ${ }^{5}$ Dr. Damodar Tiwari,MBBS. MD.Department of Paediatrics, College of Medical Sciences, Bharatpur, Chitwan, Nepal

\section{Address for correspondence: \\ Dr. Poonam Sharma \\ Registrar Department of Paediatrics, \\ Shahid Gangalal National Heart Center, \\ Kathmandu, Nepal \\ E-mail: medponam@gmail.com \\ Tel: +9779855056256}

\section{How to cite}

Sharma P, Sarmah BK, Kayastha P, Shrestha A, Tiwari D. Clinical Profile of Children with Acute Febrile Encephalopathy in a Tertiary Health Care Center of Nepal. J Nepal Paediatr Soc 2015;35(3):224-230.

doi: http://dx.doi.org/10.3126/jnps.v35i3.13882

This work is licensed under a Creative Commons Attribution 3.0 License.

\section{(c) (i)}

This diffuse and nonspecific brain insult manifested by a combination of coma, seizures and decerebration, is an important cause of morbidity and mortality in young hospitalized children ${ }^{3}$ making significant demands of intensive and high dependency care resources ${ }^{4}$.

The profile of acute febrile encephalopathy varies not only in different geographical regions of world but also in different areas and seasons in the same 
country ${ }^{3}$. Although central nervous system infection is the most common cause of fever with altered sensorium in children throughout the world ${ }^{4}$ it can result from a wide variety of primary etiologies, posing a diagnostic challenge to medical staff. Bacterial meningitis, aseptic meningitis, encephalitis, cerebral malaria, CNS tuberculosis and enteric encephalopathy are some of the common causes. ${ }^{3}$ The survivors are often left with disabling neurological problems if not diagnosed in time and aggressively managed ${ }^{1,5}$. Outcome of such illness may range from complete recovery through varying degrees of neurological disability to death from either acute ilness or complications. A better understanding of the causes and outcomes of this heterogeneous group of children will aid the design of protocols for their investigation and management ${ }^{6}$.

There is significantly more severe morbidity and higher mortality due to acute febrile encephalopathy in developing countries like Nepal where adequate health facilities may not be readily available or accessible to everyone. There is lack of proper utilization and affordability of health resources among the general population due to various adverse cultural and socioeconomic factors ${ }^{7}$. Similarly malnutrition and anemia which are rampant in developing countries have been known to be a significant contributing factor to mortality in these patients ${ }^{8}$. Proper data on incidence, severity, and outcome are a prerequisite for informed provision of health-care resources for this group ${ }^{6}$. Very limited work has been done to know the actual burden of the different neurological disease in Nepalese children?

This prospective observational study was conducted for one year to evaluate the profile of hospitalized children with acute febrile encephalopathy, to recognize the prevalence of local causes and to highlight the preventive and therapeutic intervention to help in reduction and improvement in the management of these topical diseases.

\section{Material and Methods}

This prospective observational study was done in children admitted with acute febrile encephalopathy within a span of one year (April 2011 to March 2012) in the Department of Paediatrics of College of Medical Sciences-Teaching Hospital which is a tertiary care referral hospital in Central Nepal.

Acute febrile encephalopathy was defined as fever for less than 2 weeks duration and altered sensorium (a modified GCS of less than 12 for more than 12 hours).
All hospitalized children of 1 month to 14 years of age with acute febrile encephalopathy admitted within April 2011 to March 2012 were included in the study. Children having evidence of traumatic coma, febrile convulsions, seizure disorder, hypoglycemia, hypoxia, dyselectrolemia, uremia and similar disorders were excluded from the study.

Detail systemic and neurological examination was done to identify the probable cause and to note the severity of neurological damage. Baseline investigations were done as per the hospital protocol and special investigations were done according to the suspected diagnosis. Lumber puncture was done in all cases unless contraindicated and detail CSF examination was done. Neuroimaging with CT scan brain was done as and when required or feasible.

Analysis was performed using the statistical software package SPSS version 20. Statistical method included determination of mean, standard deviation, chi-square test, $p$-value and co-relation coefficient values. This study was approved by the ethical review board of College of Medical Sciences-Teaching Hospital, Bharatpur, Nepal.

\section{Results}

Among total of 1072 patients admitted in the pediatric ward, 59 cases presented with acute onset fever with encephalopathy. However, only 54 children were included in this study as the rest either expired or went against medical advice before establishment of diagnosis. The incidence of acute febrile encephalopathy was 5.50\% among whom 19 (35.2\%) were female and 35 (64.8\%) children were male. There were 24 children (44.4\%) within the age group of one to five years. Table 1 shows the distribution of cases among different age groups.

Table 1: Distribution of cases according to the age group

\begin{tabular}{|l|c|c|}
\hline Age group & Number of children & Percent \\
\hline Less than one year & 6 & 11.1 \\
\hline 1-5 years & 24 & 44.4 \\
\hline 5-10 years & 12 & 22.2 \\
\hline 10-14 years & 12 & 22.2 \\
\hline Total & $\mathbf{5 4}$ & $\mathbf{1 0 0 . 0}$ \\
\hline
\end{tabular}

The average duration of hospital stay was 13.76 days with maximum duration of 26 days and minimum was 8 days stay. In this study 29 (53.7\%) children presented during the monsoon season while 13 
(24.1\%) children presented in the summer season and $12(22.2 \%)$ children presented in the winter. According to Kupusswamy socioeconomic scale, 32 (59.3\%) children were from lower class family while 21 (38.9\%) were from middle class and only one case was from upper class family.

The mean duration of fever was 4.41 days with fever lasting for less than 48 hours in 15 (27.8\%) children, for $2-7$ days in $33(61.1 \%)$ children and for more than seven days in six (11.1\%) children.Similarly the mean duration of onset of altered sensorium was $28 \pm 19$ hours with 38 (70.4\%) children having altered sensorium for less than 24 hours, 15 (27.8\%) children for 24 to 72 hours and one child with more than three days. The GCS was less than 7 in two cases, 7-10 in $24(44.4 \%)$ cases, $10-12$ in 20 (37\%) cases and more than 12 in eightcases. The average GCS at the time of presentation was $10.5 \pm 10.5$ with minimum of 6 and maximum of 12 .

Table 2: Distribution of presenting complains among children

\begin{tabular}{|c|c|c|}
\hline Symptoms & No. of children & Percentage \\
\hline Headache & 15 & 27.8 \\
\hline Seizure & 32 & 59.3 \\
\hline Rashes & 3 & 5.6 \\
\hline Photophobia & 5 & 9.3 \\
\hline Vomiting & 25 & 46.3 \\
\hline Others & 14 & 25.9 \\
\hline
\end{tabular}

Seizure and vomiting were the most common presenting complains seen in 32 (59.3\%) and 25 (46.3\%) children respectively. Table No. 2 shows the frequency of various presenting complains and Table 3 shows the frequency of various clinical features among children with acute febrile encephalopathy. Meningeal irritation signs were present in 27 (50\%) children among which neck stiffness was present in 25 (46.3\%), Kernig's sign in 24 (44.4\%) and Brudzinsky sign in 9 (16.7\%) children.

Viral encephalitis was the most common diagnosis seen in 34 (63\%) children followed by bacterial meningitis in 12 (22.2\%) children, cerebral malaria, enteric encephalopathy and hepatic encephalopathy in four, three and one case respectively. Among those with viral encephalitis, one case each was diagnosed as HSV and Mumps encephalitis. The maximum number of children with viral encephalitis were within 5 to 14 years of age seen in 17 (50\%) cases and those with bacterial meningitis were within 1 to 5 year of age seen in 8 (66.7\%) children. In this study, 21 (61.8\%) children with viral encephalitis presented with seizures in comparison to 7 (58.3\%) children with bacterial meningitis. Similarly 17 (50\%) cases of viral encephalitis presented with a GCS of 10 to 12 whereas 8 (66.9\%) cases of bacterial meningitis presented with a GCS of 7 to 10 . In this study meningeal signs were present in $18(52.9 \%)$ of viral encephalitis, $8(66.7 \%)$ of bacterial meningitis. CT Scan Brain was found to be abnormal in $7(13 \%)$ cases with three having cerebral edema, two having ventricular enhancement and one each of calcified granuloma and sinus thrombosis.

Table 3: Distribution of cases according to the frequency of the clinical signs

\begin{tabular}{|l|c|c|c|}
\hline Signs & & Number of children & Percentage \\
\hline Pupillary size & Abnormal & 6 & 11.1 \\
\hline Papillary reaction & Non reacting & 2 & 3.7 \\
\hline \multirow{2}{*}{ Respiratory rate } & Increased & 5 & 35.2 \\
\hline \multirow{2}{*}{ Heart rate } & Decreased & 42 & 79.3 \\
\hline \multirow{2}{*}{ Blood pressure } & Increased & 3 & 5.6 \\
\hline Rashes & Decreased & 5 & 18.5 \\
\hline Papilledema & Hypertension & 10 & 9.3 \\
\hline \multirow{2}{*}{ Tone } & Hypotension & 2 & 3.7 \\
\hline Power & Present & 7 & 13 \\
\hline \multirow{2}{*}{ Deep Tendon reflex } & Present & 13 & 24.1 \\
\hline \multirow{2}{*}{ Babinsky reflex } & Increased & 3 & 5.6 \\
\cline { 2 - 4 } & Decreased & 6 & 11.1 \\
\cline { 2 - 4 } & Decreased & 16 & 29.6 \\
\hline
\end{tabular}




\section{Discussion}

It is a known fact that acute febrile encephalopathy is an important source of morbidity and mortality among children that make heavy demands in pediatric intensive care unit and neuro-rehabilitation resources. Despite being such an important disease entity there is paucity in the studies regarding the incidence, clinical features, etiology and prognosis of this disease in the world literature. Especially in Nepal, very few studies regarding this relatively common neurologic disease have been conducted. This study has been done in order to fulfill some of the gap in the available data.

There is lack of sufficient data regarding the actual incidence of acute febrile encephalopathy in children and almost all of the data are hospital based. Wong et al. has estimated the incidence of nontraumatic coma to be $30 / 100,000$ children in the Northern region of England $^{6}$. The incidence of acute febrile encephalopathy in this hospital was $5.50 \%$ which is similar to another study done in eastern Nepal where the incidence was noted to be $6.2 \%$, however a much higher incidence of $10-15 \%$ was noted in India ${ }^{9,10} 58$ had JE. Ten patients had concomitant infections, four with malaria and six with bacterial meningitis, and were excluded from analysis. Clinical findings were as follows: boys, 69\%; age 4-14 years, 71\%; presentation during summer and autumn, 83\%; fever >3 days, 69\%; altered sensorium $<2$ days, 50\%; Glasgow coma score $8-12,63 \%$; seizures, $58 \%$. Four $(8.3 \%$. The most common age of children affected with acute febrile encephalopathy in this study was within one to five years of age which is similar to the study done in India ${ }^{10}$ and to correlate clinical and radiological features of patients with viral encephalitis. \n\nMETHODS: A prospective hospital based study conducted on the consecutive patients admitted in a pediatric unit during the period of 1 (st. However in Malaysia, febrile encephalopathy was found to occur at a much younger age with half of the cases being less than one year of age ${ }^{11}$.

Similarly male were found to be more affected than females with a ratio of $1.8: 1$ which is consistent with that noted by various authors ${ }^{2,3,10,11,12}$ pulse, heart rate, blood pressure, coma severity by Glasgow coma scale (GCS. Although no plausible mechanism has been proposed for the male preponderance of acute febrile encephalopathy, it may possibly be because of the gender biasness in seeking medical care especially in this part of the world. As noted by Khinchi et al from Nepal, this study also revealed increased number of cases during the monsoon season of July to August which may be due to the increase transmission of the diseases during the monsoon months ${ }^{13}$. Similarly acute febrile encephalopathy was found to occur more frequently in people of low socioeconomic status in our country, present in 32 (59\%) children, which was similar to that noted in India ${ }^{10}$ and to correlate clinical and radiological features of patients with viral encephalitis. \n\nMETHODS: A prospective hospital based study conducted on the consecutive patients admitted in a pediatric unit during the period of 1 (st. This could be because increased incidence of overcrowding, poor hygiene, poor nutritional status and suboptimal immunity increases susceptibility to various infectious diseases, febrile encephalopathy being one of them. One alarming fact noted during the study was only $50 \%$ of children of more than one year had received complete vaccination including the Japanese encephalitis vaccine. JE vaccines were not received by $37.5 \%$ of children and $12.5 \%$ of parents did not know about the vaccination status of their child. Japanese encephalitis vaccine has been introduced in the national immunization schedule of Nepal since 2009 initially in 16 districts extending to 31 endemic districts of the Terai region ${ }^{14}$.

In this study, the mean duration of fever was 4.4 \pm 3.57 days similar to that noted by Rayamajhi et al. ${ }^{9} 58$ had JE. Ten patients had concomitant infections, four with malaria and six with bacterial meningitis, and were excluded from analysis. Clinical findings were as follows: boys, 69\%; age 4-14 years, 71\%; presentation during summer and autumn, $83 \%$; fever $>3$ days, $69 \%$; altered sensorium $<2$ days, $50 \%$; Glasgow coma score $8-12,63 \%$; seizures, $58 \%$. Four $(8.3 \%$ The fever was present for less than 72 hours in $47.8 \%$ of children in the current study which is more than that noted by Singh et $\mathrm{al}^{3}$ from Nepal and less than that noted by Anga et al of Papua New Guinea ${ }^{2}$. In the same study made by Singh et al in a tertiary center of Eastern Nepal, the mean duration of altered sensorium and of GCS was $27.9 \pm 19.1$ hours and $9.6 \pm 3.2$ respectively with $96.3 \%$ of children having the altered sensorium for less than 72 hours which is similar to the observation made in the current study ${ }^{3}$. Similarly the median GCS of 12 in our study was similar to that made by Anga et al where the median was $13^{2}$. A negative correlation between the GCS at presentation and the time taken for normalization was being observed. This suggests that children who had a higher GCS at presentation recovered quickly in comparison to those with poorer GCS. Various authors have noted GCS at the time of presentation and the duration of altered sensorium to be a significant predictor of normal outcome ${ }^{2,9}$ defined as fever, seizures and/or altered consciousness, is a common presentation in children 
in tropical developing countries. Outcomes range from complete recovery through varying degrees of neurological disability which slowly resolve or remain permanent to death from either the acute illness or complications. Whilst bacterial meningitis accounts for a proportion of children affected, the aetiology in many remains unclear but includes malaria and probably viral encephalitis. AIM: To understand the aetiology, presentation and outcome of febrile encephalopathy in children in Papua New Guinea. METHODS: Children aged between 1 month and 12 years presenting to Port Moresby General Hospital with febrile encephalopathy were studied prospectively. A detailed history and examination and the following laboratory investigations were undertaken as appropriate: cerebrospinal fluid (CSF.

The common clinical features that a child with febrile encephalopathy presents with are seizures, vomiting and headache which could be due to the direct cerebral insult or it could also suggest increased intracranial pressure. In our study seizure was the most common symptom which was present in $59.3 \%$ which was followed by vomiting and headache. Analysis of the symptoms were done by various authors were Rayamajhi et al. and Singh et al. noticed seizures to be present in $58 \%$ and $50 \%$ of children respectively ${ }^{3,9}$ blood and CSF cultures, peripheral smear and serology for malarial parasite, and serology for Japanese encephalitis (JE. The various clinical signs and symptoms as described in Table 2 is consistent with that noted by different national and international authors ${ }^{2,3,9} 58$ had JE. Ten patients had concomitant infections, four with malaria and six with bacterial meningitis, and were excluded from analysis. Clinical findings were as follows: boys, 69\%; age 4-14 years, $71 \%$; presentation during summer and autumn, 83\%; fever $>3$ days, 69\%; altered sensorium <2 days, 50\%; Glasgow coma score 8-12, 63\%; seizures, 58\%. Four $18.3 \%$.

In the current study signs of meningeal irritation were evaluated in 46 children who were of more than two years of age among whom positive results were seen in $50 \%$ of children similar to that observed by Karmakar et al. ${ }^{10}$ and to correlate clinical and radiological features of patients with viral encephalitis. In\nMETHODS: A prospective hospital based study conducted on the consecutive patients admitted in a pediatric unit during the period of 1(st In our study, neck stiffness was present in $46.3 \%$ of children. Various authors have reported different frequencies of neck stiffness ranging from $30 \%$ to $50 \%$ of children with febrile encephalopathy ${ }^{2,12}$. Similar to our observation, authors have reported a higher incidence of Kernig's sign present in 44 to 50\% while Brudzinski sign was the least common sign of meningeal irritation ${ }^{2,3,15}$ defined as fever, seizures and/ or altered consciousness, is a common presentation in children in tropical developing countries. Outcomes range from complete recovery through varying degrees of neurological disability which slowly resolve or remain permanent to death from either the acute illness or complications. Whilst bacterial meningitis accounts for a proportion of children affected, the aetiology in many remains unclear but includes malaria and probably viral encephalitis. AIM: To understand the aetiology, presentation and outcome of febrile encephalopathy in children in Papua New Guinea. METHODS: Children aged between 1 month and 12 years presenting to Port Moresby General Hospital with febrile encephalopathy were studied prospectively. A detailed history and examination and the following laboratory investigations were undertaken as appropriate: cerebrospinal fluid (CSF.

Viral encephalitis was the most common cause of febrile encephalopathy seen in $63 \%$ of cases followed by bacterial meningitis, cerebral malaria and enteric encephalopathy seen in $22.2 \%, 7.4 \%$ and 5.6 $\%$ of cases respectively. Similar etiology was noted in Central India where acute febrile encephalopathy was most common due to viral encephalitis followed by pyogenic meningitis, cerebral malaria and tuberculosis meningitis ${ }^{16}$ prospective study, carried out in a tertiary care hospital of central India. Duration of the study was two years (2010 to 2012. Singh et al. reported viral encephalitis to be the most common illness presenting with fever and altered sensorium which was present in $45.8 \%$ of cases $^{3}$. Similarly bacterial meningitis was second most common diagnosis which was present in $42 \%$ of the cases and the cerebral malaria in $7.5 \%$ of cases. However Kumar et al. showed bacterial meningitis to be one of the predominant cause of febrile encephalopathy which was present in $44 \%$ of cases and viral encephalitis in $36.4 \%$ of cases $^{17}$ and any organisms were cultured. Blood examination included white cell count and estimations of haemoglobin, urea, glucose, and electrolyte concentrations and serum alanine aminotransferase and aspartate aminotransferase. A firm diagnosis was established in 278 patients $(38 \%$.

In the current study, among 39 cases who underwent CT scan of brain, abnormal findings were noted in $13 \%$ of the children. Among the abnormal findings, cerebral edema was the most common finding which was present in $5.5 \%$ of children followed by ventricular enhancement, calcified granuloma and 
sinus thrombosis in three, one and one case each. Nadel et al. reported abnormal CT scan finding present in $36 \%$ of the children with acute febrile encephalopathy however $5 \%$ of the cases had accidental finding ${ }^{18}$. The most common finding was cerebral edema followed by ventricular dilatation. As the finding of the CT scan are no specific and do not help in the management or in the diagnosis, emergency CT scan head in a child with acute febrile encephalopathy does not have much significance ${ }^{18}$. Its usefulness is limited to assess for signs of intracranial mass effect prior to lumbar puncture and to monitor complications like hydrocephalus ${ }^{19}$.

While comparing among viral encephalitis and bacterial meningitis, the current study noted that children with bacterial meningitis were slightly younger than those with viral encephalitis. Similarly seizure was more common in viral encephalitis whereas low GCS at presentation was common in bacterial meningitis. Similarly $52.9 \%$ of children with viral encephalitis had signs of meningeal irritation in comparison to $66.7 \%$ of cases with bacterial meningitis.This observation could have been made as bacterial meningitis has a prolonged course of illness and causes more focal and local irritation of brain and meninges in comparison to a more global involvement of the brain in viral infection. However because of small sample size this data was not statistically significant

\section{Conclusion}

Acute onset of fever with altered sensorium is a common problem encountered by a pediatrician especially those practicing in or nearby tropical countries. Found to be more common in monsoon months, inadequate nutrition and poor hygiene increased the risk while early health care seeking behavior reduced the morbidity and the possible mortality of this dreadful condition. Emergency CT scan in children with acute febrile encephalopathy has limited role for monitoring complication, hence emergency management and stabilization of the child has the prior importance.

\section{Limitations of this study}

Being a hospital based study done in a tertiary care center; the incidence observed in this study may not reflect the actual incidence of acute febrile encephalopathy of the entire population. Also because of the small sample size correlation between the individual diagnosis with different clinical features and prognostic factors could not be made. Due to the limitations in the laboratory investigations facility, identification of the causative organism could not be made in most of the case.

\section{Acknowledgement: None \\ Funding: Nil \\ Conflict of Interest: None \\ Permission from IRB: Yes}

\section{References}

1. Yeolekar ME, Trivedi TH. Febrile encephalopathy: challenges in management. J Assoc Physicians India 2006;54:845-847.

2. Anga G, Barnabas R, Kaminiel $O$, et al. The aetiology, clinical presentations and outcome of febrile encephalopathy in children in Papua New Guinea. Ann Trop Paediatr 2010;30(2):109-118.

3. Singh RR, Chaudhary SK, Bhatta NK, Khanal B, Shah D. Clinical and etiological profile of acute febrile encephalopathy in Eastern Nepal. Indian J Pediatr 2009;76(11):1109-1111.

4. Modi M, Goyal M.Acute onset Fever and Altered Sensorium: Acute Febrile Encephalopathy. In: Bhalla A (ed). Update on Tropical fever. India, Indian College of Physicians; 2015: 60-74.

5. Nayana Prabha PC, Nalini P, Tiroumourougane Serane V. Role of Glasgow Coma Scale in pediatric nontraumatic coma. Indian Pediatr 2003;40(7):620-625.

6. Wong CP, Forsyth RJ, Kelly TP, Eyre J a. Incidence, aetiology, and outcome of non-traumatic coma: a population based study. Arch Dis Child 2001;84(3):193-199.

7. Mishra N, Belbase M, Shrestha D, Poudel R, Mishra P. Childhood neurological illness in Nepal. J Nepal Health Res Counc 2010;8(1):55-62.

8. Buch PM, Palmar P, Doshi SK, Chudasama RK. Outcome predictors of NonTraumatic Coma with Infective Etiology in Children. J Pharmac and Biomed Sci 2011;12(12):1-4.

9. Rayamajhi A, Singh R, Prasad R, Khanal B, Singhi S. Clinico-laboratory profile and outcome of Japanese encephalitis in Nepali children. Ann Trop Paediatr 2006;26(4):293-301.

10. Karmarkar SA, Aneja S, Khare S, Saini A, Seth A, Chauhan BKY. A study of acute febrile encephalopathy with special reference to viral etiology. Indian J Pediatr 2008;75(8):801-805.

11. Sofiah A, Hussain IH. Childhood non-traumatic coma in Kuala Lumpur, Malaysia. Ann Trop Paediatr 1997;17(4):327-331. 
12. Bansal A, Singhi SC, Singhi PD, Khandelwal N, Ramesh S. Non traumatic coma. Indian J Pediatr 2005;72(6):467-473.

13. R, Khinchi; Y A, Kumar; S Y. Study of acute encephalitis syndrome in children. J Coll Med Sci 2010;6(1):7-13.

14. EPI Fact Sheet Nepal 2012. World Health Organization Regional Office for South East Asia. Available from: http://searo.who.int/entity/ immunization/data/nepal_epi_factsheet_2012. pdf (Accessed on 25th March 2015)

15. Rayamajhi A, Singh R, Prasad R, Khanal B, Singhi S. Study of Japanese encephalitis and other viral encephalitis in Nepali children. Pediatr Int 2007;49(6):978-984.
16. Bokade C, Gulhane R, Bagul A, Thakre S. Acute febrile encephalopathy in children and predictors of mortality. J Clin Diagn Res 2014;8(8):PC09PC11.

17. Kumar R, Mathur A, Kumar A, Sethi GD, Sharma $S$, Chaturvedi UC. Virological investigations of acute encephalopathy in India. Arch Dis Child 1990;65(11):1227-1230.

18. Nadel S, Joarder R, Gibson M, Stevens J, Britto J, Habibi P. Emergency cranial computed tomography in the management of acute febrile encephalopathy in children. J Accid Emerg Med 1999;16:403-406.

19. Dineen RA, Jaspan T. Neuroimaging in children. Curr Paediatr 2006;16(5):348-359. 